\title{
Far-lateral approach for surgical treatment of fusiform PICA aneurysm
}

\author{
William T. Couldwell, M.D., Ph.D. and Jayson A. Neil, M.D.
}

Department of Neurosurgery, Clinical Neurosciences Center, University of Utah, Salt Lake City, Utah

Ruptured fusiform posterior inferior cerebellar artery (PICA) aneurysms can be technically challenging lesions. Surgeons must be ready to employ a variety of strategies in the successful treatment of these aneurysms. Strategies include complex clip techniques including clip-wrapping or trapping and revascularization. The case presented here is of a man with subarachnoid hemorrhage from a fusiform ruptured PICA aneurysm. The technique demonstrated is a far-lateral approach and a clip-wrap technique using muslin gauze. The patient was given aspirin preoperatively in preparation for possible occipital-PICA bypass if direct repair was not feasible. It is the authors' preference to perform direct vessel repair as a primary goal and use bypass techniques when this is not possible. Vessel patency was evaluated after clipwrapping using intraoperative Doppler. Intraoperative somatosensory and motor evoked potential monitoring is used in such cases. The patient recovered well.

The video can be found here: http://youtu.be/iwLqufH47Ds.

KEY WORDS far lateral approach; aneurysm; posterior inferior cerebellar artery; fusiform; clip-wrap 